\title{
Antimigraine drugs: new frontiers
}

\author{
A. Rapoport
}

(C) The Author(s) 2009. This article is published with open access at Springerlink.com

\begin{abstract}
There are many acute care and preventive medications for the treatment of migraine. However, patients may often find that their headaches are not under optimal control. There are several targets that have been looked at and studied for the production of new, more effective medications. There are also effective devices for therapy of migraine. A list of targets will be put forth and a small number of them will be described in greater detail in this paper.
\end{abstract}

Keywords Migraine - Migraine treatment .

Botulinum toxin type A - CGRP antagonists .

Dihydroergotamine $\cdot$ Ketorolac $\cdot$ Sumatriptan

\section{Introduction}

Migraine is a chronic and at times disabling disorder, usually consisting of attacks of significant headache, various associated symptoms (i.e. nausea, vomiting, sonophobia, photophobia and worsening with exertion) and sometimes visual or other types of auras. It affects about $12 \%$ of the population of the US and other Western countries. There are several categories of acute care medications including over the counter substances and prescription medications. They include simple analgesics, combination analgesics, NSAIDs, prescription analgesics, ergots and triptans.

In Europe and Canada, the first triptan was launched in 1991. In the US, it was first available in 1993 and today

A. Rapoport $(\square)$

Department of Neurology,

The David Geffen School of Medicine at UCLA in Los Angeles,

239 South Orange Drive, Los Angeles, CA 90036, USA

e-mail: alanrapoport@gmail.com there are seven triptans available with a variety of formulations including tablets, injections, orally disintegrating tablets and nasal sprays. Although triptans are usually considered as the first-line treatment for acute care of migraine attacks, some patients cannot afford them, over one-third of patients do not respond ideally to triptans and over half are willing to try other treatments than the one they are currently taking.

There are only five preventive medications approved by the FDA and only four of them are available in the US at this time, two beta blockers and two antiepileptic medications. However, none of them works in more than $50 \%$ of patients and they all have significant possible adverse events.

There are multiple targets that form the basis for possible new acute care and preventive treatments for migraine. This paper will list many categories of them and concentrate on some new drugs which may become available within the next few years.

\section{Categories of future acute care and preventive medication for migraine}

There are many future targets and some are being investigated as possible new therapies and others have already led to the creation of drugs that are currently in clinical trials [1]. What follows is a listing of future targets:

1. Calcium channel modulators

2. Sodium channel blockers (lacosamide, a slow sodium channel blocker)

3. Glutamate blockers

4. Novel antiepileptics (SV2A blockers, e.g. levetiracetam)

5. GABA enhancers and analogs (valproate)

6. $\mathrm{AT}_{2}$ (angiotensin 2 inhibitors) 
7. Alpha-2 agonists

8. $5-\mathrm{HT}_{2 \mathrm{~A}}$ antagonists

9. $5-\mathrm{HT}_{7}$ antagonists

10. Acetylcholine receptor modulators

11. BDNF modulators (brain-derived neurotrophic factor)

12. Orexin-melatonin pathway modulators

13. Dopamine antagonists delivered by oral inhalation (prochlorperazine and loxapine)

14. Sigma receptor agonists (dextromethorphan and others)

15. Non AMPA-kainate glutamate receptor modulators

16. Potassium current modulators

17. Chloride channel enhancers

18. Connexin hemi-channel modulators (tight junction antagonists, e.g. tonabersat)

19. NOS inhibitors (nitric oxide synthase)

20. Arachidonic cascade modulators (COX-2 and cysteinyl leukotriene antagonists)

21. Astrocytic calcium wave inhibitors

22. Existing and new $5-\mathrm{HT}_{1 \mathrm{~B} / \mathrm{D} / \mathrm{F}}$ agonists

23. CGRP antagonists (olcegepant and telcagepant)

24. Glutamate modulators (memantine and tezampanel)

25. Anti-inflammatory drugs [NSAIDs: ketorolac nasal spray (ROX-828) and diclofenac (PRO-153)]

26. COX-3 inhibitors (dipyrone in Brazil)

27. Peripheral cannabinoid agonists $\left(\mathrm{CB}_{1}\right.$-dronabinol)

28. $\mathrm{TRPV}_{1}$ antagonists (+civamide-like drugs)

29. NSAIDs

30. Prostanoid antagonists

31. Others.

\section{CGRP receptor antagonists}

Calcitonin gene-related peptide, closely related structurally to calcitonin and amylin, has been intensely studied over the last 20 years as an agent possibly related to migraine pathophysiology. CGRP is involved in sensory neurotransmission and can be found in most sensory nerves, especially those trigeminovascular afferents in the meninges involved in migraine [2]. It is one of the most potent vasodilators known. CGRP levels measured in the jugular venous system are elevated after migraine and cluster headache attacks, and are normalized by therapy with sumatriptan. For years, it was thought that blocking its dilating effect might help to treat migraine and its antagonism held promise to be a novel strategy to relieve migraine headache. It is now known to effectively block migraine pain without overt vasoconstriction. If and when they gain approval in the US by the FDA and in other countries, CGRP receptor antagonists would be the first non-serotonergic, non-vasoconstricting, migraine specific medication.
CGRP has been shown to have several sites of action including blood vessels, mast cells in the meninges and as a facilitator of pain transmission the brain stem [3]. CGRP receptors have been found in trigeminal ganglion, in the brain stem in neurons of the trigeminal nucleus caudalis and in smooth muscle of the meningeal vasculature [6]. CGRP can be blocked by a fragment of the peptide containing amino acids 8-37 (CGRP 8-37). The first effective CGRP receptor blocker was BIBN4096 (olcegepant). It was reported that intravenous administration helped a significant number of patients versus placebo, without constricting blood vessels in preclinical studies [4]. Telcagepant, previously termed MK-0974, was the first reported oral formulation of a CGRP receptor antagonist. It has been reported to work well in migraine in a phase IIB study published in Neurology and recently in a phase III study published in Lancet [5, 6]. Preclinical data suggest that telcagepant is not a vasoconstrictor and clinical studies show it to be as effective as rizatriptan and zolmitriptan and as tolerable as placebo. It is predicted that this drug could be launched in 2010, possibly to be followed by one or two competitors sometime afterwards.

In the recently published article, telcagepant $300 \mathrm{mg}$ was found to be as effective as zolmitriptan with fewer adverse events [6]. This was a randomized, paralleltreatment, placebo and active-controlled, double-blind, trial performed at 81 sites in Europe and the USA of adults with migraine diagnosed by International Headache Society criteria. Patients treated moderate or severe migraine attacks with either oral telcagepant 150 or $300 \mathrm{mg}$, zolmitriptan $5 \mathrm{mg}$, or placebo. There were five co-primary endpoints: pain freedom, pain relief and absence of nausea, photophobia and phonophobia, at $2 \mathrm{~h}$ after treatment.

According to Dr. Ho's article, “1,380 patients were randomly assigned to receive telcagepant $150 \mathrm{mg}(n=$ 333), $300 \mathrm{mg}(n=354)$, zolmitriptan $5 \mathrm{mg}(n=345)$ or placebo $(n=348)$. Telcagepant $300 \mathrm{mg}$ was more effective than placebo for pain freedom (95 [27\%] of 353 patients vs. 33 [10\%] of $343[P<0.0001])$, pain relief $(194$ [55\%] of 353 vs. 95 [28\%] of $343[P<0.0001])$, and absence of phonophobia (204 [58\%] of 353 vs. 126 [37\%] of $342[P<0.0001]$ ), photophobia (180 [51\%] of 353 vs. 99 [29\%] of $342[P<0.0001])$, and nausea $(229$ [65\%] of 352 vs. 189 [55\%] of $342[P=0.0061])$. The efficacy of telcagepant $300 \mathrm{mg}$ and zolmitriptan $5 \mathrm{mg}$ were much the same, and both were more effective than telcagepant $150 \mathrm{mg}$. Adverse events were recorded for $31 \%$ taking telcagepant $150 \mathrm{mg}, 37 \%$ taking telcagepant $300 \mathrm{mg}, 51 \%$ taking zolmitriptan $5 \mathrm{mg}$, and $32 \%$ taking placebo." The measurement of $2-24 \mathrm{~h}$ sustained pain freedom was slightly better numerically for telcagepant $300 \mathrm{mg}$ versus zolmitriptan $5 \mathrm{mg}$, but there was no statistical difference. 
A potential benefit of telcagepant and other CGRP receptor antagonists is the lack of vasoconstriction in animal models. This suggests that they may be able to be given to patients with vascular disease, but that was not studied in this trial as zolmitriptan is contraindicated in patients with vascular disease and will have to be studied in the future.

\section{Transdermal patches}

Recently, sumatriptan became the first of the seven triptans to become generic in several countries, which has led to the development of generic formulations of available products and to the design of some novel products containing the generic formulation, including needle-less injection, sublingual, intranasal and patch forms. One of the most interesting products in development, which may address the unmet need of the nauseated migraineur and/or the patient who does not absorb oral medication optimally during a migraine attack, is a sumatriptan patch. NP101, from NuPathe, is an iontophoretic patch that delivers sumatriptan transdermally. It utilizes a small electric current to drive sumatriptan across the skin delivering 6 or $12 \mathrm{~mA} / \mathrm{h}$ and maintaining sumatriptan plasma levels above the target level of $\geq 10 \mathrm{ng} / \mathrm{ml}$ for greater than $7 \mathrm{~h}$ [7]. There is a linear relationship between the applied current and drug delivery. As a result, drug delivery is precisely controlled at desired levels, providing consistent therapeutic drug levels. In pK studies, the patch delivered sumatriptan more consistently than either the $100 \mathrm{mg}$ oral tablet or $20 \mathrm{mg}$ nasal preparation. This finding supports the hypothesis that parenteral administration (subcutaneous or transdermal) provides more predictable delivery by bypassing absorption through the GI tract.

At the intended plasma concentrations delivered by the patch, which were in between those of the $20 \mathrm{mg}$ nasal spray and $100 \mathrm{mg}$ oral tablet, the patches were well tolerated. No subject reported atypical pain and pressure sensations or other common triptan adverse events after application of NP101 patches. The most common adverse event for NP101 was application site-related pruritus, which was generally mild and resolved without treatment. No subject withdrew from the study due to local skin irritation. The data suggest that transdermal iontophoretic delivery of sumatriptan with NP101 may offer significant clinical utility for migraine patients, including circumventing underlying migraine-associated GI disturbances including nausea and gastric stasis. The patch also provides consistent, predictable delivery of desired drug levels over a $4 \mathrm{~h}$ period. This offers the potential to avoid atypical pain, pressure and other sensations commonly associated with current triptan formulations.

\section{Oral inhalers}

Three drugs are being tested as inhalers: DHE, prochlorperazine and loxapine. The last two are dopamine antagonists, a class of drugs that has been shown to treat migraine acutely when given intravenously. DHE (dihydroergotamine mesylate) has been available in various forms for over 50 years and still remains the mainstay of treatment at major headache centers in the US when patients require hospitalization or comes to the emergency room after having already developed central sensitization. It is usually given several times per day intravenously. It is also used orally in Europe as a preventive and intranasally in the US and Canada as an acute care medication. The intravenous preparation is often very effective, but cannot be used at home and often causes the patient to become more nauseated or even vomit, in spite of pretreatment with an antiemetic. Oral inhalation seems to provide similar efficacy with the ease of home use and fewer adverse events.

Studies were performed with a specially designed device called the Tempo Inhaler (MAP Pharma), to deliver DHE deep into the lung after breath actuation [8].

A phase I study of four doses of orally inhaled DHE delivered by the specially designed inhaler versus $1 \mathrm{mg}$ of IV DHE $(n=18)$ was performed. There was a rapid systemic absorption of DHE with a $t_{\max }$ of $12 \mathrm{~min}$ with a $0.88 \mathrm{mg}$ respirable dose (vs. a $6 \mathrm{~min} t_{\max }$ with the IV preparation). The systemic levels attained were slightly lower than with IV DHE, with the ratio of AUC 0-infinity of inhaled versus IV approximately 0.77 . The Tempo ${ }^{\circledR}$ inhaler is a proprietary, novel, breath-actuated device that is expected to deliver most of the drug to the deep lung, thereby minimizing oropharyngeal deposition. Phase II data suggest an onset of action comparable to IV administration of DHE, with relief that is both rapid and sustained [9]. Phase II results demonstrate that $32 \%$ of patients achieve pain relief as early as $10 \mathrm{~min}(P=0.019)$ at $0.5 \mathrm{mg}$ dose. This is somewhat lower that the usual dose IV.

DHE delivered by this inhaler was well tolerated in phase II studies with no serious adverse events. There was decreased nausea and no clinically significant changes observed in pulmonary function tests, clinical lab findings, heart rate, blood pressure or respiratory rate. Phase III studies are ongoing and have been designed to confirm that the onset of action is $10 \mathrm{~min}$ versus placebo, that there is sustained pain relief and freedom over 24 and $48 \mathrm{~h}$, with good safety and tolerability.

\section{Nasal sprays}

There are three nasal sprays currently in use for the acute treatment of migraine in the US, including two triptans, 
sumatriptan and zolmitriptan and DHE. Intranasal ketorolac has been studied in a convenient, single dose device in a formulation specifically designed for episodic use. It provides a $p \mathrm{~K}$ profile equivalent to that of ketorolac administered intramuscularly. Ketorolac is a racemic NSAID, which inhibits the cyclooxygenase system (COX 1 and COX 2), and hence prostaglandin synthesis, with potent analgesic and moderate anti-inflammatory activity. It is highly water soluble and can be delivered in an amount suitable for intranasal administration $(100 \mu \mathrm{l})$.

In a phase I, single dose, five-way crossover, randomized study, absorption of ketorolac started immediately, and median $t_{\max }$ ranged from 0.50 to $0.75 \mathrm{~h}$ postdose, irrespective of the dose of ketorolac [10]. There was a terminal phase half-life of approximately 5-6 h. Very similar profiles were observed for the IM doses.

Ketorolac has no active metabolites and is metabolized in the liver by glucuronidation and parahydroxylation. Further studies compared the pharmacokinetics of intranasal versus intramuscular dosing. ROX-828, from Roxro, is a nasal spray formulation of ketorolac composed of $30 \mathrm{mg}+12 \mathrm{mg}$ lidocaine, which reduces nasal irritation. The $p \mathrm{~K}$ profile is equal to or better than ketorolac given intramuscularly. The preparation utilizes a single dose device with two available sprays. The plasma concentration curves show that this intranasal formulation achieves peak blood levels faster than IM injections.

A phase II trial, done in Germany and Finland, consisted of a double-blind, placebo- controlled, randomized, single dose study using $30 \mathrm{mg}$ of ketorolac.

The primary endpoint was $2 \mathrm{~h}$ pain freedom. The $n$ was ROX-828 $=68$ and placebo $=73$. The primary end point just missed significance (if 3 outlier patients were removed, the primary endpoint was significant at $2 \mathrm{~h}$ ). Pain relief was significant at 1, 1.5, 2, 3 and $4 \mathrm{~h}$. An intranasal dose of $30 \mathrm{mg}$ produced plasma level roughly equivalent to $20 \mathrm{mg}$ intramuscularly. Further studies are planned, possibly with a higher dose.

\section{Gap junction blocker (preventive)}

Tonabersat is a novel benzoyl-aminobenzopyran anticonvulsant and antimigraine medication developed by SmithKline Beecham. It binds selectively with high affinity to a unique stereoselective site on neurons and glia. It has no affinity for any other previously established antiepilepsy or antimigraine site. Tonabersat inhibits cortical spreading depression number and duration. Unpublished studies with carabersat (a close structural analog) in a rat hippocampal slice model, demonstrated that the action of the class is related to the inhibition of neuronal-glial gap junctions. There was also inhibition of electrical coupling of
GABAergic inter-neurons. Tonabersat is $2-3$ times more potent than carabersat. Recent rat trigeminal ganglion research in vivo indicated that the effect of tonabersat on gap junctions (hemi-channel communication) is mediated by connexin 26 .

Early studies were done for acute treatment of migraine and only showed a trend for efficacy. A trial investigated the efficacy of SB-220453 in the glyceryltrinitrate (GTN) human experimental migraine model [11]. The study reported, " 15 patients with migraine without aura entered a randomized, double-blind, crossover study with $40 \mathrm{mg}$ or placebo followed by a 20-min GTN infusion. Four subjects had a hypotensive episode after SB-220453 plus GTN but none after GTN alone. The reaction was unexpected, since animal models and previous human studies had shown no vascular or sympatholytic activity with SB-220453. The study was terminated prematurely because of this interaction. SB220453 had no significant pre-emptive anti-migraine activity compared with placebo in this human model of migraine."

Tonabersat was then studied as a preventive migraine agent. According to the Minster Pharmaceuticals website, "Tonabersat completed a phase IIA clinical trial in prophylaxis (prevention) of migraine in 2007. Positive data from this study include a significant increase in subjects classed as 'responders'-defined as a $50 \%$ or greater reduction in migraine attacks at the end of the 3 months of treatment - on tonabersat compared with those on placebo. The figures were $62 \%$ for tonabersat compared with $45 \%$ for placebo $(P<0.05)$."

A double-blind, controlled, randomized clinical trial of migraine with and without aura was performed in patients on no preventive migraine medication. The doses were 20-40 mg, given once daily, for 3 months. The reduction in migraine days was 3.7 days for placebo and 4.4 days for tonabersat, which was not significant. Adverse events were generally mild and the drug was well tolerated. Fifty-one percent of placebo patients and $61 \%$ of tonabersat patients had treatment-emergent AEs. Treatment-related adverse events were $15 \%$ for placebo and $39 \%$ for tonabersat. Adverse events leading to withdrawal were two for tonabersat (nausea and dizziness) and one for placebo (dizziness and memory impairment). There were no laboratory abnormalities. Apparently, a phase IIB trial did not meet its primary endpoint as announced by Minster Pharmaceuticals in early February 2009. The future development of this migraine medication is unclear.

\section{Neurotoxin therapy: botulinum toxin injections (preventive)}

Although the exact mechanism of action of botulinum toxin type A injections as a treatment for headache are not 
known, it is thought that that the antinociceptive action is probably independent of its anti-cholinergic effects at the neuromuscular junction. It is no longer believed that the relaxation or induced weakness of muscles contributes to the therapeutic effect. Instead inhibition of peripheral sensitization, leading to the inhibition of central sensitization through the blocking of glutamate, substance $\mathrm{P}$ and CGRP peripherally, is thought to lead to the therapeutic effect. There have been many positive open trials and a few double-blind, controlled studies with conflicting reports of efficacy. In a chronic migraine trail, in which the primary endpoint was not reached, efficacy was shown only in a subgroup not taking preventive medication [12].

A recent study to compare the effectiveness of treatment of transformed migraine between botulinum toxin type A and topiramate demonstrated that both groups had significantly fewer headaches compared with baseline 6 months after the start of therapy. At 9 months, the two treatments were equivalent. More patients in the topiramate group dropped out of the study due to adverse events [13]. Another recent study compared botulinum toxin type A with divalproex in episodic and chronic migraine. The data demonstrate that both treatments showed a significant reduction in disability with fewer adverse events in the botulinum toxin type A treated group [14].

Two large, double-blind, placebo-controlled, randomized phase III trials were performed in patients with chronic migraine according to the IHS definition who were not on preventive medication. Although the results have not been released or published, a press release was issued by Allergan in September 2008 about the results [15].

The primary endpoint for the first trial was change from baseline in the number of headache episodes at the end of 3 months. In the second trial, the primary endpoint was the change in number of headache days in a 28-day period at the end of 3 months. In the second phase III study, the primary endpoint and secondary endpoint showed statistically significant benefit of botulinum toxin type A treatment over placebo injections. Patients treated with botulinum toxin type A demonstrated a greater decrease in both number of headache days $(P<0.001)$ (primary endpoint) and number of headache episodes $(P=0.003)$ (secondary endpoint). However, the first phase III study did not meet its primary endpoint. It is thought that if the FDA suggested endpoint had been used (decrease in number of headache days), the first study would also have been significant. It is suspected that Allergan will file for an indication for treatment of chronic migraine with botulinum toxin type A sometime in 2009 , based on these two phase III studies.

\section{Conclusion}

There are many new acute care and preventive therapies being investigated for the treatment of migraine. A few of them, which should be available in the near future, have been presented here. I am cautiously optimistic that some of them will make it to the clinic and will be effective additions to the headache specialist's armamentarium.

Conflict of interest statement A. Rapoport is on the Advisory Boards of NuPathe, MAP and Roxro. A. Rapoport is an author of the Phase IIB study on telcagepant.

Open Access This article is distributed under the terms of the Creative Commons Attribution Noncommercial License which permits any noncommercial use, distribution, and reproduction in any medium, provided the original author(s) and source are credited.

\section{References}

1. Ramadan NM, Buchanan TM (2006) New and future migraine therapy. Pharmacol Ther 112:199-212

2. Goadsby PJ, Edvinsson L (1993) The trigeminovascular system and migraine: studies characterizing cerebrovascular and neuropeptide changes seen in humans and cats. Ann Neurol 33: $48-56$

3. Levy D, Burstein R, Strassman AM (2005) Calcitonin generelated peptide does not excite or sensitize meningeal nociceptors: implications for the pathophysiology of migraine. Ann Neurol 58:698-705

4. Olesen J, Diener HC, Husstedt IW et al (2004) Calcitonin generelated peptide receptor antagonist BIBN 4096 BS for the acute treatment of migraine. N Engl J Med 350:1104-1110

5. Ho TW, Mannix LK, Fan X et al (2008) Randomized controlled trial of an oral CGRP receptor antagonist, MK-0974, in acute treatment of migraine. Neurology 70:1304-1312

6. Ho TW, Ferrari MD, Dodick DW et al (2008) Efficacy and tolerability of MK-0974 (telcagepant), a new oral antagonist of calcitonin gene-related peptide receptor, compared with zolmitriptan for acute migraine: a randomised, placebo-controlled, parallel-treatment trial. Lancet 372:2089-2090

7. Pierce M, Marbury T, O'Neill C et al (2008) A novel patch formulation of sumatriptan succinate utilizing SmartRelief ${ }^{\mathrm{TM}}$ transdermal technology. Data presented at the 50th annual scientific meeting of the American Headache Society 28 June 2008

8. Armer T, Shrewsbury S, Newman S et al (2007) Aerosol delivery of ergotamine tartrate via a breath-synchronized plume-control inhaler in humans. Curr Med Res Opin 23:3177-3187

9. Shrewsbury S, Cook R, Taylor G et al (2008) Safety and pharmacokinetics of dihydroergotamine mesylate administered via a novel $\left(\right.$ TEMPO $^{\circledR}$ ) Inhaler. Headache 48:355-367

10. McAleer SD, Majid O, Venables E et al (2007) Pharmacokinetics and safety of ketorolac following single intranasal and intramuscular administration in healthy volunteers. J Clin Pharmacol 47:13-18

11. Tvedskov JF, Iversen HK, Olesen J (2004) A double-blind study of SB-220453 (Tonerbasat) in the glyceryltrinitrate (GTN) model of migraine. Cephalalgia 24:875-882

12. Dodick DW, Mauskop A, Elkind AH et al (2005) Botulinum toxin type A for the prophylaxis of chronic daily headache: subgroup analysis of patients not receiving other prophylactic 
medications: a randomized double-blind, placebo-controlled study. Headache 45:315-324

13. Mathew NT (2008) A double-blind comparison of botulinum toxin type A (BoNTA) and topiramate for the prophylactic treatment of transformed migraine headaches: a pilot study. Presented at the 12th congress of the European Federation of the European Societies: 23-26 Aug 2008, Madrid, Spain
14. Blumenfeld AM, Schim JD, Chippendale TJ (2008) Botulinum toxin type A and divalproex sodium for prophylactic treatment of episodic or chronic migraine. Headache 48:210-220

15. Allergan announces positive top-line results from phase III BOTOX headache program—Released 11 Sept 20080900 a.m. Eastern Daylight Time 